\title{
Espectro clínico da infecção por COVID-19 nos organismos humanos: revisão bibliográfica
}

\author{
Clinical spectrum of COVID-19 infection in human organisms: narrative review
}

Espectro clínico de la infección COVID-19 en organismos humanos: revisión narrativa

Francisco Clezion Franca Vasconcelos Júnior ${ }^{1 *}$, Alba Angélica Nunes Mouta ${ }^{2}$, Ravena Vasconcelos Aragão $^{3}$, Giovanna Stefanne Lópes Barbosa ${ }^{2}$, Priscila Favoritto Lopes ${ }^{2}$, Augusto César Beltrão da Silva ${ }^{4}$, Brisa Fidelis Gândara ${ }^{2}$, Bruna Caroline Ribeiro Beltrão ${ }^{4}$, Demétrio Félix Beltrão da Silva ${ }^{4}$, Renata Paula Lima Beltrão ${ }^{2,4}$.

\begin{abstract}
RESUMO
Objetivo: Analisar através de uma revisão as apresentações clínicas mais relevantes da infecção pelo SARSCoV-2. Revisão Bibliográfica: O COVID-19 possui um espectro amplo de apresentações. A forma assintomática é um desafio diagnóstico, levando à subnotificação. Acredita-se que ocorram em indivíduos com eficiente defesa antiviral e resposta imune efetiva, mas incapazes de bloquear completamente o vírus. A Síndrome Gripal Leve representa $80 \%$ dos casos sintomáticos, manifestados através de febre, tosse, fadiga, dispneia e mialgia. Os casos graves, de $5-10 \%$ dos sintomáticos, geralmente se expressam por pneumonia, com alterações de sinais vitais, dispneia ou disfunção orgânica, além disso, podem evoluir para uma Síndrome do Desconforto Respiratório Agudo, com complicações respiratórias, alterações radiográficas e da PaO2/FiO2. A Sepse e Choque séptico por COVID-19 possuem ainda maior chance de desfecho desfavorável, condições que indicam permanência do paciente em ambiente de terapia intensiva. Considerações Finais: Pouco ainda se sabe sobre o COVID-19, mas seu alto potencial de transmissibilidade e sua variabilidade de apresentações clínicas são reconhecidos como fatores complicadores do controle da epidemia.
\end{abstract}

Palavras-chave: Infecções por coronavírus, Sinais e sintomas, Sintomas gerais, Exacerbação dos sintomas, Humanos.

\begin{abstract}
Objective: To analyze through a review the most relevant clinical presentations of SARS-CoV-2 infection. Bibliographic Review: COVID-19 has a wide spectrum of presentations. The asymptomatic form is a diagnostic challenge, leading to underreporting. They are believed to occur in individuals with efficient antiviral defense and effective immune response, but unable to completely block the virus. Mild Flu Syndrome represents $80 \%$ of symptomatic cases, manifested through fever, cough, fatigue, dyspnoea and myalgia. Severe cases, of $5-10 \%$ of those symptomatic, are usually expressed by pneumonia, with changes in vital signs, dyspnea or organ dysfunction, in addition, they may progress to an Acute Respiratory Discomfort Syndrome, with respiratory complications, radiographic and $\mathrm{PaO} 2$ / FiO2 changes. Sepsis and septic shock by COVID-19 have an even greater chance of an unfavorable outcome, conditions that indicate the patient's stay in an intensive care environment. Final Considerations: Little is known about COVID-19, but its high potential for transmissibility and its variability in clinical presentations are recognized as complicating factors in controlling the epidemic.
\end{abstract}

Key words: Coronavirus infections, Signs and symptoms, General symptoms, Symptom exacerbation, Human.

\footnotetext{
1 Universidade Federal do Ceará (UFC). Sobral - Ceará.

*E-mail: clezionjr@gmail.com

2 Universidade Federal do Delta do Parnaíba (UFDPar). Parnaíba - Piauí.

${ }^{3}$ Centro Universitário UNINTA (UNINTA). Sobral - Ceará.

${ }^{4}$ Instituto de Ensino Superior do Vale do Parnaíba (IESVAP). Parnaíba - Piauí.
} 


\section{RESUMEN}

Objetivo: Analizar mediante una revisión las presentaciones clínicas más relevantes de la infección por SARS-CoV-2. Revisión bibliográfica: COVID-19 tiene un amplio espectro de presentaciones. La forma asintomática es un desafío diagnóstico, lo que lleva a un subregistro. Se cree que ocurren en individuos con defensa antiviral eficiente y una respuesta inmune efectiva, pero que no pueden bloquear completamente el virus. El síndrome de la gripe ligera representa el $80 \%$ de los casos sintomáticos, que se manifiestan a través de fiebre, tos, fatiga, disnea y mialgia. Los casos graves, del 5 al $10 \%$ de los sintomáticos, generalmente se expresan por neumonía, con cambios en los signos vitales, disnea o disfunción orgânica, además, pueden progresar a un Síndrome de incomodidad respiratoria aguda, con complicaciones respiratorias, cambios radiográficos y de PaO2 / FiO2. La sepsis y el shock séptico por COVID-19 tienen una probabilidad aún mayor de un resultado desfavorable, condiciones que indican la estadía del paciente en un entorno de cuidados intensivos. Consideraciones finales: Poco se sabe sobre COVID-19, pero su alto potencial de transmisibilidad y su variabilidad en las presentaciones clínicas se reconocen como factores que complican el control de la epidemia.

Palabras clave: Infecciones por coronavirus, Signos y síntomas, Síntomas generales, Exacerbación de los síntomas, Humanos.

\section{INTRODUÇÃO}

O COVID-19 (Corona Vírus Disease 2019), denominação dada pela Organização Mundial da Saúde (OMS), é uma infecção causada pelo vírus SARS-CoV-2 da família dos coronavírus, constituído por uma fita simples de RNA. Esse vírus é semelhante aos vírus da Síndrome Aguda Respiratória Grave (SARS) e da Síndrome Aguda Respiratória Grave do Oriente Médio (MERS), que assolaram a China em 2002 e 2003 e o Oriente Médio em 2012, respectivamente. Porém, a transmissibilidade do SARS-CoV-2 é mais elevada, tornando a disseminação dez vezes maior que a do SARS-CoV (COSTA IBSS, et al., 2020). Devido a isso, a doença que se originou inicialmente em dezembro de 2019 na cidade chinesa de Wuhan, já se tornou uma pandemia que preocupa a população mundial há 3 meses, em decorrência dos mais de 700 mil casos confirmados e dos mais de 30 mil óbitos (WUJTEWICZ MA, et al., 2020).

A infecção ocorre por meio de gotículas de saliva expelidas no ar ou pelo contato com pessoas contaminadas. Ainda há poucas informações sobre o vírus, mas sabe-se que ele tem alta transmissibilidade e provoca casos leves, em torno de $80 \%$, a casos mais graves com insuficiência respiratória, em torno de 5 a $10 \%$. Os sintomas da doença são febre acima de $37,8^{\circ} \mathrm{C}$, tosse seca, fadiga, dispneia, mialgia e, com menos frequência, diarreia e dor de garganta (BRASIL, 2020a).

A insuficiência respiratória da forma crítica da doença faz parte de uma Síndrome do Desconforto Respiratório Agudo (SDRA) decorrente de lesões pulmonares causadas pelo SARS-CoV-2, e que se manifesta principalmente por dispneia de graus leves a intensos, tornando necessário suporte ventilatório em Unidades de Terapia Intensiva (UTI) e outros cuidados que evitem complicações mais graves, como pneumonias e sepse (YUAN M, et al., 2020). O COVID-19 causou uma enorme mobilização mundial acerca da tomada de práticas preventivas para tentar combater a propagação viral, pois estudos mostraram que pacientes assintomáticos podem transmitir a doença durante o período de incubação que é de 2 a 14 dias, antes mesmo de apresentarem os sintomas (SINGHAL T, 2020).

O diagnóstico do COVID-19 leva em conta o exame físico do paciente, mas também a investigação clínicoepidemiológica. Daí a importância de indagar sobre viagens internacionais recentes, contato com pessoas que viajaram ou com casos sabidamente confirmados com COVID-19. Inicialmente, a doença se manifesta com síndrome gripal, podendo progredir com aumento da febre por 3 a 4 dias, tosse seca e dispneia leve a intensa, o que difere da infecção pelo vírus Influenza. O diagnóstico laboratorial é feito com técnicas de RTPCR e sequenciamento do genoma viral (BRASIL, 2020b).

O tratamento consiste, inicialmente, da triagem e identificação de fatores de risco, a internação, oxigenoterapia e técnicas de ventilação podem ser considerados em pacientes com dispneia severa com quadro de SDRA. O tratamento sintomático e medidas de suporte podem ser usados para controle da febre, 
tosse, dores ou vômitos, se houver. Pode-se lançar mão de anticolinérgicos para pacientes com dispneia, tosse, sibilos ou SDRA e anticoagulantes para pacientes com disfunção de coagulação. $O$ tratamento farmacológico específico ainda é controverso, há na literatura evidências de benefícios de antimaláricos (cloroquina e hidroxicloroquina), antibioticoterapia (azitromicina e outros), antivirais (lopinavir/ritonavir, remdesivir e umifenovir), ivermectina, dentre outros. Porém, ainda há poucos estudos que evidenciem a efetividade desses fármacos no tratamento em larga escala para os diversos perfis de pacientes (BRASIL, 2020b).

Embora diversos estudos clínicos estejam sendo desenvolvidos de forma acelerada no esforço de encontrar vacinas ou tratamentos efetivos, não se chegou a um consenso de como tratar a doença, nem se sabe por quanto tempo perdurará a pandemia. Na China, a taxa geral de mortalidade já atingiu 2,3\%, superando a taxa da gripe sazonal. A maioria dessas mortes foram de paciente idosos, acima de 60 anos, e comorbidades subjacentes, como cardiopatias, Hipertensão Arterial Sistêmica e o Diabetes Mellitus, neoplasias e doenças respiratórias crônicas (FEDSON DS, et al., 2020).

O COVID-19 pode ocasionar complicações como pneumonia, podendo ser quadros mais leves ou graves, nesse último caso evoluem com hipóxia ou com taxas de até $50 \%$ de acometimento pulmonar em exames de imagem e até mesmo choque séptico e disfunção de órgãos. Enquanto estudos são realizados, o espectro clínico da doença desafia a população mundial no combate às diversas formas de manifestação da infecção (MCINTOSH MD, et al., 2020)

Diante disso, o presente artigo tem como objetivo analisar através de uma revisão narrativa as síndromes clínicas mais relevantes associadas à infecção pelo COVID-19, de forma a observar como a doença se manifesta nos organismos humanos a partir de uma revisão de estudos atuais sobre o tema.

\section{REVISÃO BIBLIOGRÁFICA}

\section{Quadro assintomático}

A existência de pacientes assintomáticos é um enorme desafio para controle da infecção, pois LI R, et al. (2020) trouxe por meio de um modelo matemático que 85\% das transmissões da COVID-19 ocorrem por pacientes assintomáticos. Fases pré-sintomáticas mostraram um fator vital na determinação do pico de casos da infecção e no sucesso do controle de surtos, em decorrência da relação entre viremia e o início do quadro assintomático. $O$ estudo indicou a necessidade de um aumento na testagem e detecção de pacientes assintomáticos infectados pelo vírus e o isolamento de infecções não documentadas atualmente para controlar totalmente o SARS-CoV2.

O impasse epidemiológico vem da capacidade de transmissão mesmo na ausência de sinais e sintomatologias clínicas, dificultado medidas de bloqueio e contribuindo para a subnotificação dos casos (FUNG SY, et al., 2020). Teoricamente, quadros assintomáticos do COVID-19 podem surgir quando os pacientes apresentam defesa antiviral forte, gerando uma resposta imune efetiva, porém limitada, não sendo capaz de bloquear completamente a ação viral.

XU XW et al. (2020) defendem que tais pacientes, além de serem raros, possuem uma menor carga viral, com replicação substancialmente reduzida em comparação com aqueles que apresentam a sintomatologia típica da doença. Ou seja, a transmissibilidade destes doentes acontece, porém menor em relação aos sintomáticos.

Sendo assim, levando em consideração a transmissão da COVID-19 por pacientes na fase pré-sintomática ou mesmo daqueles assintomáticos, Lee JK e Jeong HW (2020) chamam atenção para a necessidade do distanciamento social, lavagem adequada das mãos e utilização de máscaras faciais. Como exemplo a ser seguido ainda citam o caso de um hospital universitário na Coreia do Sul que optou por, logo no início do surto no país, reduzir o número de entradas desnecessárias ao prédio, verificação da temperatura de todos os visitantes ao hospital e a utilização de mascaras, bem como lavagem adequada das mãos para todos aqueles que entrassem no hospital, independentemente da presença de sintomas. 
Os casos assintomáticos do COVID-19 são mais prevalentes em neonatos, lactentes e crianças, os quais na maioria das vezes evoluem apenas com quadro sintomático leve, apresentando apenas tosse e febre (SINGHAL T, 2020).

Segundo estudo realizado por Cao Q et al. (2020), a contagem de glóbulos brancos e absoluta de linfócitos eram, em geral, normais no grupo de crianças chinesas infectadas analisadas. Por esses motivos, crianças podem se tornar o principal disseminador do COVID-19, o que justificaria a necessidade de fechamento temporário da escola para conter a propagação da infecção.

\section{Síndrome Gripal Leve}

Do espectro clínico do COVID-19, uma Síndrome Gripal Leve perfaz a maioria dos casos sintomáticos e das manifestações clínicas da infecção pelo SARS-CoV-2, apenas uma minoria evolui rapidamente para um estado crítico com Síndrome da Angústia Respiratória Aguda, insuficiência respiratória, falência múltipla de órgãos e até mesmo óbito (LAKE MA, 2020, GHO YR, et al., 2020).

Em estudo feito por Adhikari SP et al. (2020) com a análise de revisões bibliográficas feitas a partir de casos da infecção na China, foram relatados como os sintomas mais comuns: febre, tosse, mialgia ou fadiga, enquanto sintomas menos comuns relatados incluem dor de cabeça, diarreia, hemoptise, coriza e tosse produtiva. Tal estudo avaliou ainda o tempo decorrido até a recuperação dos pacientes, sendo de 7 dias na maioria dos casos (ADHIKARI SP, et al., 2020).

Um estudo feito por Guan WJ, et al. (2020) com 1099 casos confirmados laboratorialmente, dois quais 926 apresentavam quadro não grave, expôs dados semelhantes, quantificando em porcentagem a prevalência de cada sintoma, sendo febre $(88,7 \%)$, tosse $(67,8 \%)$ e fadiga $(38,1 \%)$. Outros sintomas como falta de ar, dor de garganta e dor de cabeça representaram 18,6\%, 13,9\% e 13,6\%, respectivamente. Em análise feita por Singual T (2020), a conjuntivite também foi descrita em alguns poucos casos como um sintoma da infecção pelo SARS-CoV-2. Percebe-se, portanto, que tal sintomatologia é indistinguível de outras infecções respiratórias.

A Síndrome Gripal Leve é a principal evolução de quadros assintomáticos da infecção em neonatos, lactentes e crianças, sendo relatada como significativamente mais suave em comparação à população adulta. Tais pacientes apresentam ótimo prognóstico, alcançando a cura entre 1 e 2 semanas após início dos sintomas. A sintomatologia amena nesta parcela da população pode ser explicada, em parte, pela prática de imunização, com a vacina Bacilo de Calmette-Guérin (BCG), que parece agir no que se conhece como "imunidade treinada" (CAO Q, et al., 2020; SINGHAL T, 2020).

Kui L, et al. (2020) citaram em seus estudos que alguns dos pacientes com COVID-19 e que tinham manifestações leves de uma Síndrome Gripal apresentavam contagem normal ou diminuída de glóbulos branco e uma redução dos linfócitos, ou seja, linfopenia, sem outras alterações significativas.

Especificidades devem ser consideradas ao analisar a Síndrome Gripal Leve em determinados pacientes. Indivíduos com extremos de idades, obesos maiores de 40 anos, gestantes, pacientes com pneumopatias, cardiopatias, nefropatias, hepatopatias ou também imunossuprimidos e portadores de outros distúrbios têm uma maior chance de evoluir para quadros mais graves da doença, devido a diversos fatores que perpassam déficits de defesa do sistema imune, agravadores de afecções respiratórias que a infecção causa ou problemas metabólicos que somatizam os efeitos patogênicos do COVID-19 no organismo humano (BRASIL, 2020c).

\section{Quadro com Pneumonia e/ou SDRA}

Em um subconjunto de pacientes, até o final da primeira semana de infecção, a doença pode evoluir para um quadro de pneumonia leve a intensa. Esta progressão está associada ao aumento exacerbado de citocinas inflamatórias, incluindo IL2 (Interleucina 2), IL7 (Interleucina 7), IL10 (Interleucina 10), GCSF (Fator estimulador de colônias de granulócitos), IP10 (Proteína Indutível 10), MCP1 (Proteína Quimiotática de Monócitos tipo 1), MIP1A (Proteína Inflamatória Macrocitária tipo 1 alfa) e TNFa (Fator de Necrose Tumoral Alfa). 
A média de tempo entre o início dos sintomas até o desenvolvimento de dispneia em pacientes chineses foi de 5 dias, com $25 \%$ a $30 \%$ dos pacientes necessitando de admissão em unidades de terapia intensiva. A maioria dos pacientes afetados eram idosos com comorbidades adjacentes, havendo apenas um caso de pneumonia grave em crianças (SINGHAL T, 2020).

A pneumonia que surge em quadros graves do COVID-19 pode ser não-complicada ou grave. A pneumonia não-complicada é uma infecção de vias aéreas inferiores que difere da pneumonia complicada por não apresentar sinais de gravidade como: frequência respiratória maior de 30 incursões por minuto, dispneia, saturação de oxigênio no sangue menor que $90 \%$ em ar ambiente, cianose ou disfunção orgânica. As complicações mais comuns entre pacientes com pneumonia advêm de uma SDRA, que consiste em agravamento dos sintomas respiratórios com alterações radiológicas, edema pulmonar ou relação $\mathrm{PaO} 2 / \mathrm{FiO} 2$ $\leq 300 \mathrm{mmHg}$, além de terem desfechos como RNAemia e lesão cardíaca aguda (BRASIL, 2020c, HUANG C, et al., 2020).

Segundo Costa IBSS, et al. (2020), essas lesões cardíacas agudas provocadas pelo COVID-19 advêm do fato de que o vírus causa diversas manifestações no sistema cardiovascular como injúria miocárdica, Insuficiência Cardíaca, Síndrome de Takotsubo, choque, miocardite e arritmias. Tais manifestações parecem ter múltiplas causas, podendo ser desencadeados por descontroles entre alta demanda do metabolismo e baixa reserva cardíaca quanto de inflamação sistêmica e trombogênese. Pacientes que apresentam risco cardiovascular por Hipertensão Arterial Sistêmica, Diabetes e idade avançada ou outras doenças prévias têm mais chance de sofrerem com a resposta inflamatória gerada pela infecção viral que leva à lesão do sistema cardiovascular e do pulmão com aumento de Dímero-D, procalcitonina, proteína C reativa, troponina, NTproBNP e ferritina, podendo levar ao óbito.

Um estudo feito pelo Centro Chinês de Controle e Prevenção de Doenças mostrou o poder de letalidade dessas comorbidades e da idade avançada no desfecho fatal de pacientes. Analisou-se 44.672 casos confirmados do COVID-19, havendo 2,3\% de mortalidade. Ao buscar o perfil dessas vítimas, se evidenciou que as comorbidades mais frequentes eram hipertensão arterial, diabetes mellitus, doença cardiovascular e idade acima de 70 anos (STRABELLI TMV, UIP DE, 2020).

Em estudo chinês, realizado por Huang C, et al. (2020), evidenciou que a grande maioria dos pacientes admitidos em UTI com quadro de SRDA por COVID-19 apresentavam em estudo tomográfico pulmonar opacidades em vidro fosco bilateralmente, com acometimento periférico e central na maioria dos casos, além de envolvimento da zona inferior.

Guo HR, et al. (2020) também descreveu essas opacidades em vidro fosco em $56,4 \%$ dos pacientes analisados e uma sombra irregular bilateral em 51,8\%. Chung M et al. (2020) ao analisar pacientes internados no Hospital da Universidade de Sun Yat-Sen, citou que tais sombras tem uma morfologia arredondada e se evidenciam na periferia dos pulmões. Além dessas alterações, de forma rara foi detectado linfonodomegalia, derrame pleural e consolidações isoladas (YUAN M, et al., 2020).

Levando em conta os achados tomográficos de pacientes analisados no estudo de Guan WJ, et al. (2020), dos quais haviam $926 \mathrm{com}$ quadros não graves e $173 \mathrm{com}$ graves, foi evidenciado opacidade em vidro fosco bilateralmente em $48,5 \%$ dos não graves e $58,4 \%$ dos graves, sombreamento irregular local em $34,2 \%$ dos não graves e $53,2 \%$ dos graves, sombreamento irregular bilateral em $39,7 \%$ dos não graves e $79,2 \%$ dos graves e anormalidades intersticiais em $10,7 \%$ dos não graves e $25,4 \%$ dos graves.

Alguns estudos trazem também a presença do sinal do "Halo Invertido" em pacientes com quadro de pneumonia pelo COVID-19 pela Tomografia Computadorizada. Esse sinal consiste em áreas pulmonares com o padrão em vidro fosco já descrita, mas circundada, parcial ou totalmente, por um anel de consolidação pulmonar. Se observa em pacientes com apresentação sintomática mais tardia, podendo ter a explicação na relação entre a fisiopatologia e o processo de organização da doença. (FARIAS LPG, et al., 2020). Lake MA (2020) traz que algumas alterações laboratoriais em pacientes graves com esse tipo de acometimento do COVID-19 cursaram com o aumento substancial de protrombina e D-dímero, quando relacionados aos de cursam com quadros mais amenos (LAKE MA, 2020). 
Alguns adultos com COVID-19 mostraram uma redução abrupta ou progressiva do número absoluto de linfócitos sanguíneos no estágio inicial da doença, sendo a relação neutrófilos / linfócitos um indicador precoce e confiável para o desenvolvimento de complicações, sugerindo que o consumo de linfócitos seja resultante da rápida replicação viral. Isso levaria, muitas vezes, ao desenvolvimento de síndrome do desconforto respiratório agudo (SDRA), um quadro fatal de lesão pulmonar (CAO Q, et al., 2020).

O estudo de Guo HR, et al. (2020) foi concordante com os de Lake MA (2020) e CAO Q, et al. (2020) ao relatar em seus estudos que em pacientes graves houve um aumento de D-dímero, neutrófilos, uréia sanguínea e creatinina, além de linfopenia. Fez relações ainda acerca do estado imunológico desses pacientes ao evidenciar aumento de fatores inflamatórios IL-6 e IL-10. Em pacientes de UTI, havia níveis plasmáticos mais elevados de IL-2, IL-7, IL-10, PCM-1, MIP-1 $\alpha$ e TNF- $\alpha$.

É importante salientar que por meio de um estudo feito por Chan JFW, et al., 2020 que analisou 6 pacientes com COVID-19 com quadros de pneumonia sem Síndrome da Angústia Respiratória Aguda, que todas alterações descritas estiveram presentes entre os pacientes, o que mostra que tais evidências laboratoriais e de imagem podem estar presentes antes da evolução para uma síndrome respiratória mais grave. Além disso, $50 \%$ apresentaram alterações ainda não citadas como lactato desidrogenase e fibrinogênio elevados.

Segundo Liu Y, et al. (2020), a carga viral é crucial na determinação da gravidade da doença. Além disso, altos níveis de carga viral podem estar associados a um alto risco de miocardite fulminante. Portanto, as combinações de hipoalbuminemia, linfopenia e altas concentrações de PCR (Proteína C reativa) e LDH (Lactato Desidrogenase) em pacientes infectados com COVID-19 na admissão hospitalar podem prever lesão pulmonar aguda mais grave (LIU Y, et al., 2020).

Quadros de linfopenia e aumento de citocinas, o que é chamado de "tempestade de citocinas" na literatura, também estão presentes na Síndrome Respiratória do Oriente Médio (MERS) e na Síndrome Respiratória Aguda Grave (SARS), predizendo que tais quadros têm grande importância na patogênese do COVID-19 ou SARS-CoV-2. Alguns estudos trazem ainda que essa "tempestade de citocinas" pode desencadear quadros de sepse viral e lesão inflamatória pulmonar induzida, com consequências como pneumonite, choque, falência de órgãos e morte (NICHOLLS JM, et al., 2003, WONG CK, et al., 2004).

\section{Sepse e Choque Séptico}

Além da pneumonia aguda e da SDRA, sepse e choque séptico são condições que indicam permanência do paciente em ambiente de UTI pelo COVID-19. Estudos mostram que quadros críticos com disfunções orgânicas ou choque equivalem a $5 \%$ dos casos totais e a maioria dos pacientes submetidos a tais condições são do sexo masculino com idade média de 63 anos e com história de doenças crônicas (MCINTOSH MD, et al., 2020; WUJTEWICZ MA, et al., 2020). A sepse em adultos é uma disfunção orgânica com risco de vida que é deflagrada por uma resposta imune desregulada do hospedeiro, no caso o vírus SARS-CoV-2. Os sinais são: estado mental alterado, respiração difícil ou rápida, baixa saturação de oxigênio, redução da produção de urina, ritmo cardíaco acelerado, pulso fraco, extremidades frias ou pressão arterial baixa, manchas na pele ou evidência laboratorial de coagulopatia, trombocitopenia, acidose, lactato alto ou hiperbilirrubinemia (SINGER M, et al., 2016).

O Choque Séptico em adultos é caracterizado por hipotensão persistente, apesar da reposição volêmica, o que se exige vasopressores para manter pressão arterial média (PAM) maior ou igual a $65 \mathrm{mmHg}$ e nível de lactato sérico maior de 2 mmoL. Tal complicação equivaleu a $9 \%$ dos casos de COVID-19 na cidade chinesa de Wuhan (WANG D, et al., 2020; WUJTEWICZ MA, et al., 2020).

Chen N, et al. (2020), por meio de estudo que analisou 99 pacientes chineses de Wuhan diagnosticados com COVID-19 e que apresentavam quadros de pneumonia, expôs a letalidade da doença por desfechos como a sepse e o choque séptico. Do total de pacientes, 17\% evoluíram com uma Síndrome do Desconforto Respiratório Agudo, dos quais $64,7 \%$ foram a óbito, tendo a sepse e o choque séptico uma representatividade de $36 \%$ desses óbitos. 
Estudos mostram que desfechos como sepse ou choque séptico em pacientes com COVID-19 grave podem ser explicados por evidências laboratoriais de uma resposta inflamatória exuberante, parecida com a síndrome de liberação de citocinas, com febre persistente, marcadores inflamatórios elevados e citocinas próinflamatórias elevadas, contribuindo para a associação a doenças críticas e fatais (CUI Y, et al., 2020).

\section{CONSIDERAÇÕES FINAIS}

Ainda existe pouco conhecimento sobre a SARS-CoV2, no entanto, é de conhecimento da comunidade científica o seu alto potencial de transmissão e seus quadros clínicos variáveis, com expressões de elevada morbimortalidade. Dessa forma, o estudo foi de grande relevância para entender os diversos quadros clínicos que podem estar relacionados ao COVID-19 já que tal conhecimento possibilita relacionar ou excluir possíveis diagnósticos diferenciais e assim tornar o diagnóstico preciso e resolutivo, além do fato de que reconhecer a variedade sintomatológica e os diversos desfechos clínicos ocasionados. Por fim, deve-se atentar à importância epidemiológica que os quadros assintomáticos podem ter no processo de contágio e da tomada de medidas preventivas orientadas por órgãos de saúde mundiais.

\section{REFERÊNCIAS}

1. ADHIKAR SP, et al. Epidemiology, causes, clinical manifestation and diagnosis, prevention and control of coronavirus disease (COVID-19) during the early outbreak period: a scoping review. Infectious Diseases of Poverty. 2020; 9(29).

2. BRASIL. Ministério da Saúde. Secretaria de Atenção Especializada à Saúde Departamento de Atenção Hospitalar, Domiciliar e de Urgência. Protocolo de Tratamento do Novo Coronavírus (2019-nCoV). Brasília, 2020a.

3. BRASIL. Ministério da Saúde. Secretaria de Atenção Primária à Saúde (SAPS). Protocolo de manejo clínico do coronavírus (covid-19) na atenção primária à saúde. Brasília, 2020b.

4. BRASIL. Ministério da Saúde. Secretaria de Ciência, Tecnologia, Inovação e Insumos Estratégicos em Saúde. Departamento de Gestão e Incorporação de Tecnologias e Inovação em Saúde. Diretrizes para diagnóstico e tratamento da COVID-19. Brasília, 2020c.

5. $\mathrm{CAO} Q$, et al. SARS-CoV-2 infection in children: Transmission dynamics and clinical characteristics. Jornal da Associação Médica Formosan. 2020; 119:670-673.

6. CHAN JFW, et al. A familial cluster of pneumonia associated with the 2019 novel coronavirus indicating person-toperson transmission: a study of a family cluster. China. Lancet, 2020; 395.

7. CHEN N, et al. Epidemiological and clinical characteristics of 99 cases of 2019 novel coronavirus pneumonia in Wuhan, China: a descriptive study. China. Lancet, 2020; 395: 507-13.

8. CHUNG M, et al. CT imaging features of 2019 novel coronavirus (2019-nCoV). China. Radiology, 2020.

9. COSTA IBSS, et al. O Coração e a COVID-19: O que o Cardiologista Precisa Saber. Brasil. Arq Bras Cardiol, 2020.

10. CUI Y, et al. A 55-Day-Old Female Infant infected with COVID 19: presenting with pneumonia, liver injury, and heart damage. J Infect Dis. 2020.

11. FARIAS LPG, et al. Pneumonia por COVID-19 e o sinal do halo invertido. Brasil. J Bras Pneumol. 2020;46(2).

12. FEDSON DS, et al. Hiding in Plain Sight: an Approach to Treating Patients with Severe COVID-19 Infection. American society of microbiology. 2020; $11(2)$.

13. FUNG SY, et al. A tug-of-war between severe acute respiratory syndrome coronavirus 2 and host antiviral defence: lessons from other pathogenic viruses. Emerging Microbes \& Infections 2020; 9.

14. GUAN WJ, et al. Clinical characteristics of 2019 novel coronavirus infection in China. China. New England Journal of Medicine, 2020.

15. GUO HR, et al. The origin, transmission and clinical therapies on coronavirus disease 2019 (COVID-19) outbreak an update on the status. China. Military Medical Research, 2020; 7:11.

16. HUANG C, et al. Clinical features of patients infected with 2019 novel coronavirus in Wuhan, China. The lancet. 2020; 395.

17. KUI L, et al. Clinical characteristics of novel coronavirus cases in tertiary hospitals in Hubei Province. China. Chin Med J., 2020.

18. LAKE MA. What we know so far: COVID-19 current clinical knowledge and research. Clinical Medicine. 2020; 20(2): 124-7.

19. LEE JK, JEONG HW. Wearing facemasks regardless of symptoms is crucial for preventing spread of COVID-19 in hospitals. Infection Control \& Hospital Epidemiology, 2020: 1-4. 
20. LI R, et al. Substantial undocumented infection facilitates the rapid dissemination of novel coronavirus (SARS-CoV2). Science. 2020.

21. LIU Y, et al. Clinical and biochemical indexes from 2019-nCoV infected patients linked to viral loads and lung injury. Sci China Life Sci. 2020; 63(3).

22. MCINTOSH K, et al. Coronavirus disease 2019 (COVID-19). Uptodate. 2020.

23. NICHOLLS JM, et al. Lung pathology of fatal severe acute respiratory syndrome. Lancet, 2003; 361:1773-8.

24. SINGER M, et al. The Third International Consensus Definitions for Sepsis and Septic Shock (Sepsis-3). JAMA. 2016;315(8):801-810.

25. SINGHA T. A Review of Coronavirus Disease-2019 (COVID-19). The Indian Journal of Pediatrics. 2020; 87(4):281286.

26. STRABELLI TMV, UIP DE. COVID-19 e o Coração. Brasil. Arq Bras Cardiol, 2020.

27. WANG D, et al. Clinical characteristics of 138 hospitalized patients with 2019 novel coronavirus-infected pneumonia in Wuhan, China. JAMA. 2020.

28. WONG CK, et al. Plasma inflammatory cytokines and chemokines in severe acute respiratory syndrome. China. Clin Exp Immunol, 2004;136:95-103.

29. WUJTEWICZ MA, et al. COVID-19 - what should anaethesiologists and intensivists know about it? Anaesthesiol Intensive Ther. 2020; 52(1): 34-41.

30. XU XW, et al. Clinical findings in a group of patients infected with the 2019 novel coronavirus (SARS-Cov-2) outside of Wuhan, China: retrospective case series. 2020;368.

31. YUAN M, et al. Association of radiologic findings with mortality of patients infected with 2019 novel coronavirus in Wuhan, China. Plos one. 2020. 